\title{
The impact on risk-factor analysis of different mortality outcomes in COPD patients
}

\author{
A. Faustini, C. Marino, D. D'Ippoliti, F. Forastiere, V. Belleudi and C.A. Perucci
}

ABSTRACT: The aim of the present study was to analyse the role of potential selection processes and their impact when evaluating risk factors for 30-day mortality among patients hospitalised for chronic obstructive pulmonary disease (COPD).

A cohort of 26,039 patients aged $\geqslant 35$ yrs and hospitalised with COPD were enrolled. A 30-day follow-up was carried out using both the cause mortality register (CMR) and the hospital discharge register (HDR). Individual and hospital factors associated with 30-day mortality were studied using both mortality outcomes.

The 30 -day mortality rate was $1.21 \cdot 1,000$ patient-days $^{-1}(95 \%$ confidence interval (CI) 1.14-1.29) using the CMR, and 1.06.1,000 patient-days ${ }^{-1}$ (95\% $\left.\mathrm{Cl} 0.98-1.13\right)$ using the HDR. Male patients, the most poorly educated, those who resided outside Rome and those who had more than one hospitalisation in the previous 2 yrs were more likely to die after discharge than when hospitalised. The most frequent cause of in-hospital death was respiratory disease and after discharge, heart disease. Older age, male sex, comorbidities, previous hospitalisations for respiratory failure, and admission to a ward not appropriate to treat respiratory diseases were the most important predictors of 30-day mortality.

Using in-hospital 30-day mortality provides a significantly different estimate of the role of specific risk factors.

KEYWORDS: 30-day mortality for COPD, mortality follow-up, mortality underestimate, selection impact on outcome studies

hort-term mortality, i.e. mortality within 30 days after hospital admission, is the most frequently used outcome to evaluate hospital care quality and to study factors associated with death in patients with chronic obstructive pulmonary disease (COPD) [1, 2]. The underlying assumptions for this approach are that: 1) short-term mortality is influenced by the quality of treatment the patients receive in the hospital [3]; 2) a 30-day interval is an adequate amount of time to analyse the association of hospital treatment with short-term mortality; and 3) in-hospital 30-day mortality is a valid approximation of total 30-day mortality.

Some criticisms have emerged regarding the use of in-hospital mortality, as it is only a proxy measure of short-term outcomes and it may cause a selection of patients who are discharged before 30 days. A recent study reported a total 30-day mortality of $18.4 \%$ compared with $8.8 \%$ in-hospital mortality in patients with acute myocardial infarction (AMI) [4]; moreover, the hospital performance measures were correlated with the former but not with the latter outcome. Another study has shown an inhospital mortality of 21.2 deaths per 100,000 injured patients and a total mortality of 35.4 deaths per 100,000 injured patients [5]. The injured patients who died in hospital also presented with different characteristics than those who died within 30 days: they were younger, had more severe injuries and fewer pre-existing medical conditions. Finally, the classification of hospital quality based on both inhospital and total 30-day mortality often differ [6], suggesting the two measures are similar but not equal. Despite these limitations, in-hospital mortality is still proposed as a hospital quality indicator for AMI, congestive heart failure, stroke, gastrointestinal haemorrhage, hip fracture and pneumonia [3]. Conversely, both mortality measures have been recommend in the UK as a30-day mortality indicator of hospital performance for stroke [7].

Little is known about the impact that a possible selection of discharged patients may have in analysing factors associated with short-term out-

\section{AFFILIATION}

Dept of Epidemiology, Rome E Local Health Agency, Rome, Italy.

CORRESPONDENCE

A. Faustini

Dept of Epidemiology

LHA RME

V. S. Costanza n 53

00198 Rome

Italy

Fax: 39683060463

E-mail faustini@asplazio.it

Received:

May 162007

Accepted after revision:

April 142008

STATEMENT OF INTEREST

None declared. 
comes among COPD patients. To analyse this possible impact, the present study measured 30-day mortality from hospital admission in a cohort of COPD patients, using both the causal mortality register (CMR) and the hospital discharge records (HDR). The factors associated with the two mortality measurements were analysed.

\section{METHODS}

\section{Study population}

The study population was enrolled from the HDR and were residents of the Lazio region of Italy ( $\sim 5$ million inhabitants) aged $\geqslant 35$ yrs and hospitalised for COPD between January 1, 1998, and November 1, 2000. The subjects were identified by the primary diagnosis (International Classification of Diseases 9th revision (ICD-9) 490.X, 491.X, 492.X, 494.X and 496.X [8]) according to the information reported in the HDR. When more than one hospitalisation occurred in the study period, the first was chosen as the index hospitalisation. The clinical history of each patient was reconstructed based on hospital diagnoses from the previous 2 yrs.

\section{Follow-up and mortality estimates}

Mortality rates were followed-up for 30 days after admission using both the regional CMR and the HDR by means of an individual record-linkage; deaths reported in the HDR were identified for the index or subsequent hospitalisations. Followup started on the first day of the index hospitalisation; when the patient was discharged from another hospital on the same or previous day with a diagnosis of COPD (primary or secondary), follow-up started on the first day of the previous hospitalisation. Patients not listed in the CMR or in the HDR were assumed to be alive at the end of the study period.

For residents of Rome (Italy), vital status from each register was validated through information from the municipality register. The characteristics and underlying causes of death of patients who died in hospital and those who died after discharge were compared. Mortality rates were calculated as the number of deaths within 30 days after admission reported in each register over 1,000 patient-days; 95\% confidence intervals (CI) were calculated according to a binomial distribution.

\section{Factors associated with 30-day mortality and data analysis}

Age, sex, place of residence (Rome versus residence anywhere else in the region) and years of education were analysed. Information on smoking habits and occupation were not available.

The admission ward was selected as an indicator of care quality [3]. The hospital ward was categorised as appropriate for COPD treatment (pneumology and intensive care units (ICU)), acceptable (general medicine, geriatrics and casualty) or unsatisfactory (all others).

To account for the severity of patients' conditions at the time of hospitalisation, information about comorbidities was obtained from the previous 2 yrs' hospital records for: lung cancer and pneumoconiosis (ICD-9: 162, 505-506) [9]; chronic heart failure (ICD-9: 428) [1]; bronchopneumonia (ICD-9: 480-486) [10, 11]; pulmonary embolism (ICD-9: 415.1) [12]; respiratory failure (ICD-9: 518.8, 518.5, 786.0); kidney failure (ICD-9: 585) [13]; and all cancers apart from lung cancer (ICD-9: 585, 140-239).
Arrhythmias were added as a possible complication of $\beta$-agonists in COPD treatment [11]. The conditions tested that were associated with risk of death in COPD patients, such as vascular and heart disease other than AMI and heart failure (ICD-9: 390-459, excluding 410, 415 and 428), central nervous system diseases (ICD-9: 320-349), traumas (ICD-9: 800-829) and poisonings (ICD-9: 960-979), were included in the analysis. The methodological indications of CHARLSON et al. [14], as modified by DEYO et al. [15], were followed in the present analysis.

The number of comorbidities, the number of hospitalisations that occurred in the previous 2 yrs and those with a diagnosis of respiratory failure, were used as quantitative indicators of patient condition severity. These indicators were included in the multivariable analysis one at a time due to the high colinearity between them.

The count of person-days of observation started on the first day of hospitalisation and ended with death on the 30th day. Survival analysis was carried out. The follow-up time (days) was divided into four intervals; the grouping was the result of a segmental regression analysis [16] of the relationship between daily mortality and follow-up time that identified the lowest mortality level on day 7. Poisson regression analysis was conducted to estimate relative risk of dying and 95\% CI in univariable, as well as in multivariable, analysis as mortality rates differed in the follow-up intervals.

\section{RESULTS}

\section{0-day mortality}

Among 26,039 patients aged $\geqslant 35$ yrs who were hospitalised with COPD between 1998 and 2000, 925 deaths (3.6\% (95\% CI $3.3-3.8 \%)$ ) occurred within 30 days after admission according to the CMR; the mortality rate was $1.21 \cdot 1,000$ patient-days $^{-1}$ (95\% CI 1.14-1.29). The follow-up carried out with the HDR detected 812 deaths $(3.1 \%(2.9-3.3 \%))$; the mortality rate was $1.06 \cdot 1,000$ patient-days ${ }^{-1}(0.98-1.13)$. Both total and in-hospital mortality showed a similar decreasing trend in the follow-up period, but rates decreased more rapidly in the HDR follow-up (fig. 1). In total, 62 in-hospital deaths were not listed in the

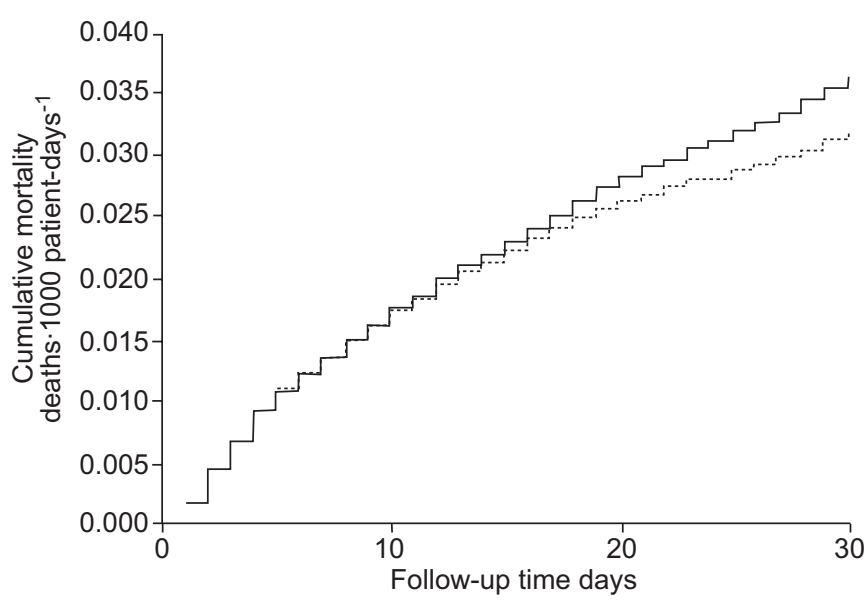

FIGURE 1. Cumulative mortality after 30-days follow-up. —_ follow-up by cause mortality register; .......... follow-up by hospital discharge register. 

TABLE 1 Risk of dying within 30 days of admission in chronic obstructive pulmonary disease patients according to two follow-up
sources

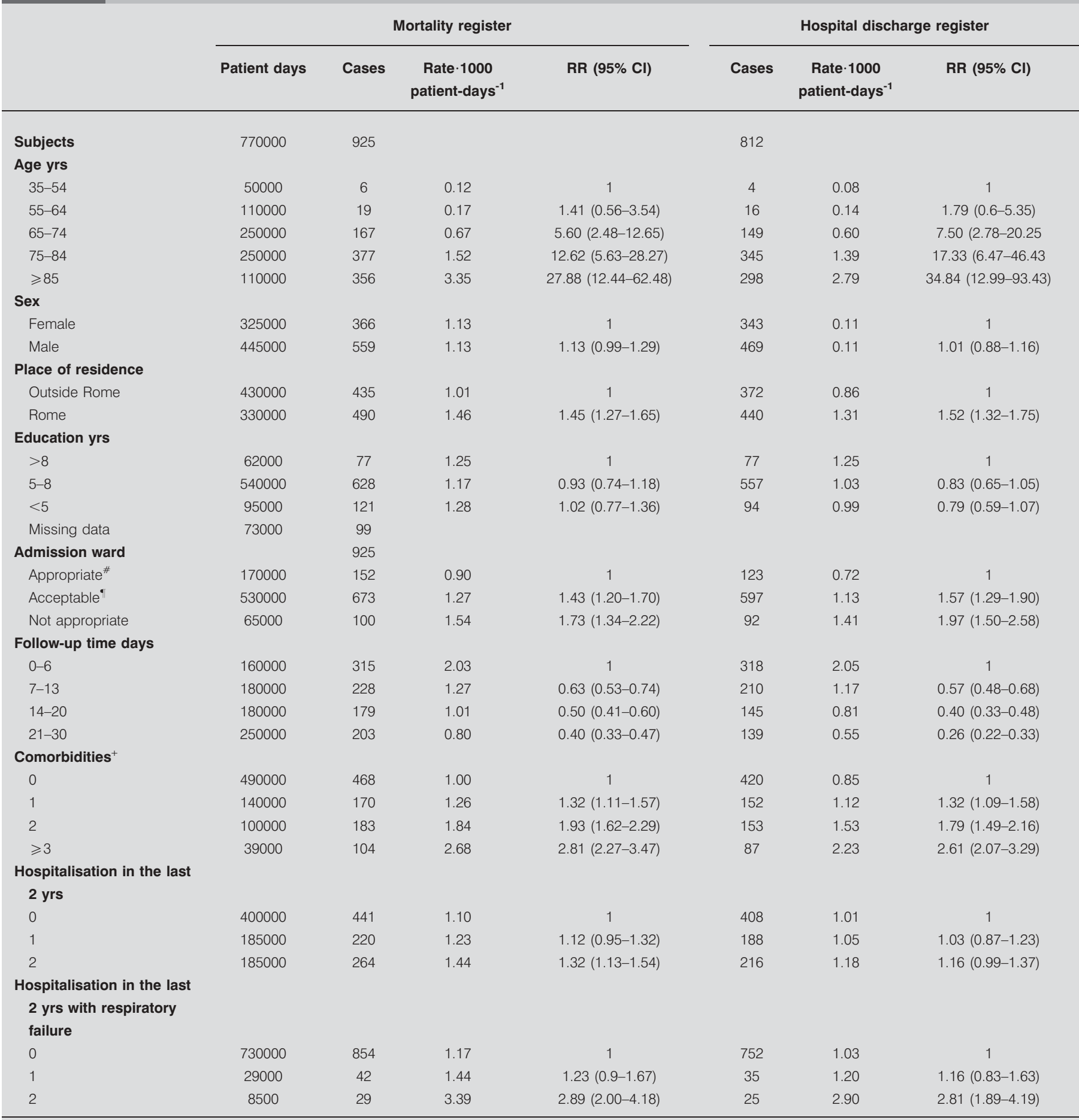

Data are presented as $\mathrm{n}$, unless otherwise stated. RR: rate ratio; $\mathrm{Cl}$ : confidence interval. ${ }^{*}$ : pneumology and intensive care unit; ": general medicine, geriatrics, causality ward; ${ }^{+}$: respiratory failure, lung cancer, pneumoconiosis, other cancer, heart failure, arrhythmias, other heart and vascular diseases excluding acute myocardial infarction, chronic kidney failure, central nervous system diseases, traumas and poisonings during previous hospitalisation.

CMR, and 175 were deaths not reported in the HDR. Only 17\% of patients were hospitalised more than once and $0.3 \%$ of them died.
Information on vital status was validated for 11,433 patients who were confirmed residents of Rome. Vital status as reported in the CMR had a sensitivity of $93.5 \%$ and a specificity 
TABLE 2 Association between various factors and 30-day mortality in chronic obstructive pulmonary disease patients according to two follow-up sources

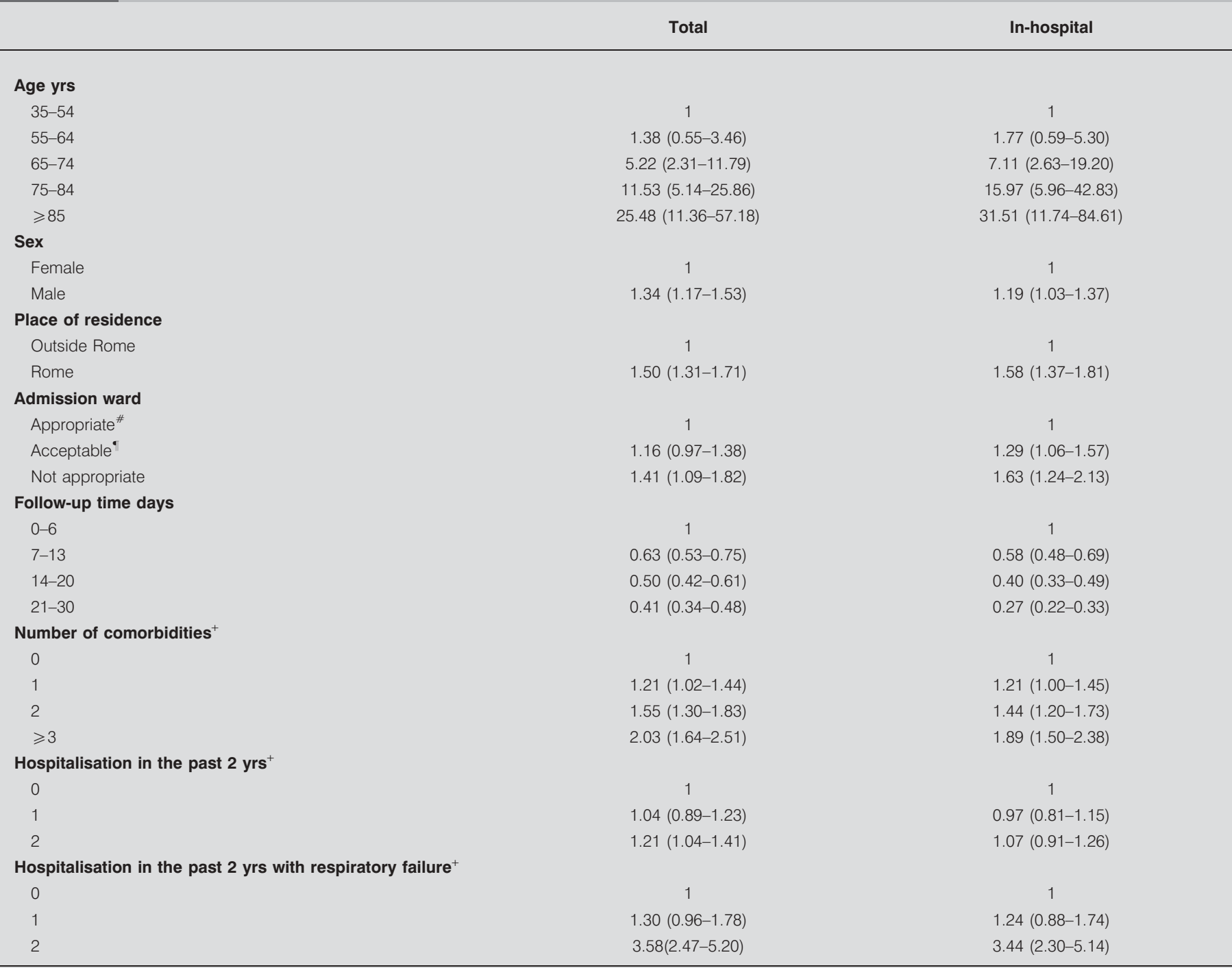

Data are presented as adjusted rate ratio (95\% confidence interval). ${ }^{*}$ : pneumology and intensive care unit; ": general medicine, geriatrics, causality ward; ${ }^{+}$: these variables were estimated in different models due to the colinearity with the last variable. The results for all other factors were obtained from the model that includes the number of comorbidities.

of $99.97 \%$; 38 patients who died according to the municipal register were not reported in the CMR, while three patients who were listed as dead in the CMR were still alive 30 days after admission. Vital status from the HDR had a sensitivity of $72.8 \%$ and a specificity of $99.99 \%$.

\section{Factors associated with 30-day mortality}

When factors associated with total 30-day mortality in 925 subjects were analysed, age $>64$ yrs, male sex, residency in Rome, being admitted to a ward other than pneumology or ICU, having at least one comorbidity or having been hospitalised at least twice in the previous 2 yrs were all associated with increased mortality (table 1). The two quantitative indicators of patient severity predicted an increased risk of death proportional to the number of comorbidities and previous hospitalisations. (table 1). The most frequent comorbidities reported in previous hospitalisations were chronic heart failure $(7 \%)$, respiratory failure $(9 \%)$, and vascular and heart diseases other than AMI and chronic heart failure (27\%); however, lung cancer (odds ratio 2.17 (95\% CI 1.25-3.75)), central nervous system diseases (2.06 (1.55$2.74))$ and renal failure $(1.98(1.29-3.04))$ were the strongest predictors of short-term mortality.

The multivariable analysis confirmed these results. However, the strength of the association was higher for sex and previous hospitalisation for respiratory failure, but weaker for age and comorbidities (table 2).

The factors associated with in-hospital mortality were very similar to those observed for total short-term mortality, but a few differences were evident in terms of effect estimates for 


\begin{tabular}{|c|c|c|c|}
\hline TABLE 3 & \multicolumn{3}{|c|}{$\begin{array}{l}\text { Comparison of the characteristics of chronic obstructive pulmonary disease patients who died in hospital or after } \\
\text { discharge within } 30 \text { days of admission }\end{array}$} \\
\hline Characteristics & Died in hospital ${ }^{\#}$ & Died after discharge & p-value \\
\hline Subjects $n$ & 750 & 125 & \\
\hline \multicolumn{4}{|l|}{ Age yrs } \\
\hline $35-54$ & 0.5 & 1.1 & 0.4 \\
\hline $75-84$ & 42.0 & 35.4 & 0.1 \\
\hline$>85$ & 37.7 & 41.7 & 0.3 \\
\hline Males & 58.3 & 69.7 & $0.005^{f}$ \\
\hline Lived in Rome & 54.5 & 46.3 & $0.05^{f}$ \\
\hline \multicolumn{4}{|l|}{ Education yrs } \\
\hline Appropriate $^{+}$ & 15.5 & 20.6 & 0.1 \\
\hline Acceptable $^{\S}$ & 73.5 & 69.7 & 0.3 \\
\hline Not appropriate & 11.1 & 9.7 & 0.6 \\
\hline \multicolumn{4}{|c|}{ Number of comorbidities } \\
\hline 0 & 51.1 & 48.6 & 0.6 \\
\hline 1 & 10.2 & 14.9 & 0.2 \\
\hline 2 & 18.9 & 23.4 & 0.2 \\
\hline$>3$ & 10.8 & 13.4 & 0.4 \\
\hline \multicolumn{4}{|c|}{ Hospitalisation in the past 2 yrs } \\
\hline 0 & 49.5 & 40.0 & $0.02^{f}$ \\
\hline 1 & 23.6 & 24.6 & 0.8 \\
\hline
\end{tabular}

Data are presented as \%, unless otherwise stated. ${ }^{\#}: 62$ patients who died in hospital and were not reported in the cause mortality register were not included; ": from a

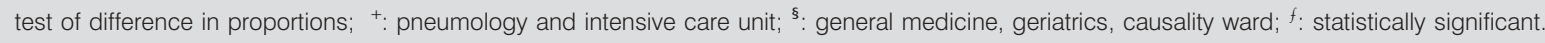

age, sex, education, residence and previous hospitalisations. A statistically significant association was not identified for sex, while the role of comorbidities became much more important in patients with more than two comorbidities and the risk of dying for residents in Rome increased. The mortality rate was the highest for the most poorly educated patients in total shortterm mortality analysis and was the lowest based on HDR data (tables 1 and 2).

\section{Characteristics of patients who died}

Table 3 shows the characteristics of the 750 patients who died in hospital and the 125 who died after hospital discharge; the 68 patients reported as dead by HDR only were not included in the comparison. Male patients, those who resided outside Rome, patients with the lowest education and those who had more than one hospitalisation in the previous 2 yrs were more likely to die after discharge than when hospitalised. In contrast, patients living in Rome, those who were well educated and those who had been not hospitalised in the previous 2 yrs were more likely to die in hospital than after discharge. No differences were observed for age, admission ward and comorbidities (table 3).

Several differences were found in the underlying causes of death for patients who died when hospitalised and those who died after discharge (table 4). COPD and all respiratory diseases were the most frequent causes of deaths for the former group of patients, while the most frequent causes of deaths were heart diseases, cancer and other vascular diseases for the latter group. Cerebrovascular diseases followed as the most frequent cause of death in both groups.

\section{DISCUSSION}

In-hospital, 30-day mortality (3.1\%) observed in the present study was consistent with estimates reported in the literature for hospitalised COPD patients. HoLGUIN et al. [17] reported mortality of $5.9 \%$ and PATIL et al. [18] observed $2.5 \%$ of deaths among patients with exacerbated COPD [18]; higher rates (8\%) were reported for acute exacerbations of COPD [2] and in 


\begin{tabular}{|c|c|c|c|c|}
\hline \multirow[t]{2}{*}{ TABLE 4} & \multicolumn{4}{|c|}{$\begin{array}{l}\text { Underlying causes of deaths in chronic } \\
\text { obstructive pulmonary disease (COPD) patients } \\
\text { who died in hospital or after discharge within } 30 \\
\text { days of admission }\end{array}$} \\
\hline & & $\begin{array}{l}\text { Died in } \\
\text { hospital }^{\#}\end{array}$ & $\begin{array}{l}\text { Died after } \\
\text { discharge }\end{array}$ & p-value \\
\hline \multicolumn{2}{|l|}{ Subjects } & $750(100)$ & $175(100)$ & \\
\hline \multicolumn{2}{|c|}{ Respiratory diseases } & $438(58.40)$ & $56(32.00)$ & 0.001 \\
\hline \multicolumn{2}{|l|}{ COPD } & $387(51.60)$ & $48(27.43)$ & 0.001 \\
\hline \multicolumn{2}{|c|}{ Respiratory failure } & $9(1.20)$ & 0 & \\
\hline \multicolumn{2}{|c|}{ Bronchopneumonia } & $9(1.20)$ & $3(1.71)$ & 0.6 \\
\hline \multicolumn{2}{|l|}{ Other } & $33(4.40)$ & $5(2.86)$ & 0.40 \\
\hline \multicolumn{2}{|l|}{ Cancer } & $35(4.67)$ & $18(10.29)$ & 0.007 \\
\hline \multicolumn{2}{|l|}{ Lung } & $1(0.13)$ & $5(2.86)$ & 0.001 \\
\hline \multicolumn{2}{|l|}{ Other } & $34(4.53)$ & $13(7.43)$ & 0.11 \\
\hline \multicolumn{2}{|l|}{ Diabetes } & $12(1.60)$ & $6(3.43)$ & 0.1 \\
\hline \multicolumn{2}{|c|}{ CNS diseases } & $8(1.07)$ & $1(0.57)$ & 0.5 \\
\hline \multicolumn{2}{|c|}{ Heart diseases } & $136(18.13)$ & 60 (34.29) & 0.001 \\
\hline \multicolumn{2}{|c|}{ Cerebrovascular diseases $^{+}$} & $34(4.53)$ & $11(6.29)$ & 0.3 \\
\hline \multicolumn{2}{|c|}{ Other vascular diseases $\$$} & $14(1.87)$ & $9(5.14)$ & 0.01 \\
\hline \multicolumn{2}{|c|}{ Digestive system diseases } & $24(3.20)$ & $6(3.43)$ & 0.8 \\
\hline \multicolumn{2}{|c|}{ Trauma/poisonings } & $13(1.73)$ & $1(0.57)$ & 0.3 \\
\hline \multicolumn{2}{|l|}{ Others ${ }^{f}$} & $16(2.13)$ & $4(2.29)$ & 0.9 \\
\hline \multicolumn{2}{|c|}{ Not well defined } & $17(2.27)$ & $2(1.14)$ & 0.3 \\
\hline \multicolumn{2}{|l|}{ Not reported } & $3(0.40)$ & $1(0.57)$ & 0.8 \\
\hline
\end{tabular}

Data are presented as n (\%), unless otherwise stated. CNS: central nervous system. ${ }^{\#}: 62$ patients who died in hospital and were not reported in the CM register were not included; " $"$ : from a test of difference in proportions; ${ }^{+}$: includes ictus and atherosclerosis; ; : includes pulmonary embolism and acute or chronic cor pulmonale; ${ }^{f}$ : includes viral hepatitis, sepsis, meningitis, metabolic diseases other than diabetes, anaemia, psychiatric diseases, urinary system diseases and decubitus ulcers

patients with respiratory failure admitted to ICU (36.6\%) [19]. However, in-hospital mortality is lower than total mortality $(3.8 \%)$ in the present study and underestimates true mortality over the follow-up time.

The differences observed between patients who died in hospital and those who died after discharge were clinically justified in most cases. COPD patients who had suffered from respiratory failure in the last 2 yrs were more likely to die in hospital during the first week [18]. The higher proportion of patients discharged in the second week of hospitalisation who died (37.1 versus $28.3 \%$ who died in hospital during the second week) could be explained in view of other observations. The mortality rate for patients discharged after the first week was, however, lower than for patients retained in hospital for $>1$ week; this suggests the patients completed the treatment, according to the expected duration of COPD exacerbation from 7 to 10 days on average. Cardiovascular diseases were the most frequent cause of death among discharged patients, and patients who died at home were more likely to have comorbidities and to have been hospitalised in the previous 2 yrs than patients who died when hospitalised, apart from hospitalisation with respiratory diseases. It could be hypothesised that patients who were discharged in the second week of hospitalisation were more likely to die from heart disease because cardiac complications occurred in these patients after discharge, although it cannot be excluded that cardiovascular problems may not have been adequately recognised during hospitalisation.

Other factors influenced a selective discharge of the patients. Male patients died more frequently at home, as did poorly educated patients. It is difficult to hypothesise the reason in both cases. Also, the patients who lived in Rome were more likely to die in the hospital than after discharge; one hypothesis could be that in Rome, comorbidities are more likely to be detected causing the patients to remain in hospital longer, since, in Rome, a higher proportion of patients were observed with comorbidities (54 versus $44 \%$ outside Rome) and a longer length of hospital stay (12.0 versus $10.3 \%$ outside Rome).

Although all of these results need further confirmation, it can be concluded that in the present data, male patients, those with the least education, those who resided outside Rome, those discharged in the second week and those who were more seriously ill with conditions other than respiratory diseases were more likely to be discharged before death and, therefore, these deaths would obviously not be reported in the HDR. The impact this selection had on the associations studied, when these characteristics are included among the risk factors, will now be discussed.

\section{Factors associated with short-term mortality}

Among the factors found to be associated with total 30-day mortality in COPD patients, being $>64$ yrs of age and male have already been reported [20], as has the presence of concomitant diseases [9-13]. The increasing risk proportional to the number of comorbidities and to the number of previous hospitalisations has been less frequently reported; however, the exposure-effect relationship observed in the current study indicates, with some confidence, that these severity indicators are true risk factors for 30-day mortality. The higher risk for residents of Rome, a large city (2.5 million inhabitants) with significant air pollution problems, suggests a role of air pollution in increasing the risk of short-term mortality in COPD patients, as previously reported [21]. The accuracy of the mortality data may be different in Rome with respect to other areas.

With regard to the indicators of care quality, in the absence of data about hospital organisation, the present authors can only hypothesise that the higher risk of death associated with wards other than pneumology, ICU and general medicine, are explained by the therapeutic protocols or the availability of adequate treatment. Comparing mortality between patients who died in hospital with those who died at home in different periods of the follow-up, the most relevant results are the higher mortality rate observed for patients who were hospitalised for only 1 week regardless of the place of death, and the higher proportion of patients who died at home than in hospital in week 2 of the follow-up. These results suggest a different risk profile for the two groups of patients; those who died in hospital in the first week were possibly too seriously ill to benefit from hospital treatment, while those who died at home in week 2 of follow-up possibly incurred treatment sideeffects or complications not recognised during admission. 
Other studies have pointed to different factors influencing mortality, including the volume of procedures [22]. The current authors did not conduct this analysis because there was not enough evidence for an association between the number of patients and the outcomes for COPD [23].

\section{Impact of using different short-term mortality measures on risk-factor analysis}

The factors associated with in-hospital mortality were very similar to those observed for total short-term mortality, with a few important differences.

A selection of patients discharged before 30 days is the most probable explanation of most differences. The consequences of this selection are missing important associations, such as the increased risk for males, exaggerating the risk for residents of Rome and lowering the risk for having comorbidities and for having been hospitalised in the previous 2 yrs for conditions other than respiratory diseases.

\section{Study limitations}

The lack of information on other determinants of mortality in COPD patients, such as occupational exposure [24] and smoking [25], could have affected the accuracy of the present study. The lack of information regarding ventilation and the date the complications began enabled the current authors to analyse the role of these factors as possible risk factors in a prospective approach for the mortality observed later. Another problem is related to the method used to assess comorbidities. Although the method is widely employed, evaluating patient history for only 2 previous years may have misclassified patients with chronic underlying diseases.

\section{Conclusion}

In conclusion, in-hospital 30-day mortality is an underestimate of total 30 -day mortality. It includes only $86.5 \%$ of all deaths and causes a selection of patients for sex, education level, residency and underlying condition severity. It was found that admission to wards that are not appropriate to treat respiratory diseases is the most important factor related to care management that is associated with 30-day mortality for chronic obstructive pulmonary disease patients. Older age, male sex and condition severity are the strongest risk factors among the individual characteristics. The present authors believe that it is vital to check for possible selection of discharged patients when in-hospital 30-day mortality is used as the preferred outcome in evaluating the quality of hospital care.

\section{ACKNOWLEDGEMENTS}

The authors would like to thank M. Becker (Dept of Epidemiology, Local Health Unit RME, Rome, Italy) for English revision and S. Pennisi (Lazio Region Public Health Agency, Rome) for help with the figure.

\section{REFERENCES}

1 Almagro P, Calbo E, Achoa de Echaguen A, et al. Mortality after hospitalization for COPD. Chest 2002; 121: 1441-1448.

2 Groenewegen KH, Schols AM, Wouters EF. Mortality and mortality-related factors after hospitalization for acute exacerbation of COPD. Chest 2003; 124: 459-467.
3 AHRQ. Guide to Inpatient Quality Indicators: Quality of Care in Hospitals - Volume, Mortality and Utilization. Publication number 02-R0204. Department of Health and Human Services, Agency for Healthcare Research and Quality, 2002.

4 Bradley EH, Herrin J, Elbel B, et al. Hospital quality or acute myocardial infarction. Correlation among process measures and relationship with short-term mortality. JAMA 2006; 296: 72-78.

5 Mullins RJ, Mann NC, Hedges JR, et al. Adequacy of hospital discharge status as a measure of outcome among injured patients. JAMA 1998; 279: 1727-1731.

6 Rosenthal GE, Baker DW, Norris DG, Way LE, Harper DL, Snow RJ. Relationship between in-hospital and 30-day standardized mortality: implications from profiling hospitals. Health Serv Res 2000; 34: 1449-1468.

7 NHS Performance Indicators: February 2002. www. performance.doh.gov.uk/nhsperformanceindicators/2002/ ha_intro.html Date last accessed: May 16, 2008.

8 Europa. European Commission for Public health. IMCA II - Indicators for Monitoring COPD and Asthma in the EU. http:/ / ec.europa.eu/health/ph_projects/2005/action1/ action1_2005_22_en.htm Date last accessed: May 16, 2007.

9 Mannino DM, Brown C, Giovino GA. Obstructive lung disease deaths in the United States from 1979 through 1993. Am J Respir Crit Care Med 1997; 156: 814-818.

10 Hansell AL, Walk JA, Soriano JB. What do chronic obstructive pulmonary disease patients die from? A multiple cause coding analysis. Eur Respir J 2003; 22: 809-814.

11 Salpeter SR, Ormiston TM, Salpeter EE. Cardiovascular effects of $\beta$-agonists in patients with asthma and COPD. Chest 2004; 125: 2309-2321.

12 Zielinski J, McNee W, Wedzicha J, et al. Causes of death in patients with COPD and chronic respiratory failure. Monaldi Arch Chest Dis 1997; 52: 43-47.

13 Antonelli Incalzi R, Fuso L, De Rosa M, et al. Co-morbidity contributes to predict mortality of patients with chronic obstructive pulmonary disease. Eur Respir J 1997; 10: 2794-2800.

14 Charlson ME, Pompei P, Ales KL, MacKenzie CR. A new method of classifying prognostic comorbidity in longitudinal studies: development and validation. J Chron Dis 1987; 40: 373-383.

15 Deyo R, Cherkin DC, Ciol MA. Adapting a clinical comorbidity index for use with ICD-9-CM administrative databases. J Clin Epidemiol 1992; 45: 613-619.

16 Muggeo VMR. Estimating regression models with unknown break-points. Stat Med 2003; 22: 3055-3071.

17 Holguin F, Folk E, Redd SC, Mannino DM. Comorbidity and mortality in COPD-related hospitalizations in the United States, 1979 to 2001. Chest 2005; 128: 2005-2011.

18 Patil SP, Krishnan JA, Lechtzin N, Diette GB. In-hospital mortality following acute exacerbations of chronic obstructive pulmonary disease. Arch Intern Med 2003; 163: 1180-1186.

19 Khilnani GC, Banga A, Sharma SK. Predictors of mortality of patients with acute respiratory failure secondary to chronic obstructive pulmonary disease admitted to an intensive care unit. A one year study. BMC Pulmonary Medicine 2004; 4: 12. 
20 Mannino DM, Homa DM, Akimbami MD, Ford ES, Redd SC. Chronic obstructive pulmonary disease surveillance, United States, 1971-2000. MMWR Surveill Summary 2002; 51: 1-16.

21 Sunyer J. Urban air pollution and chronic obstructive pulmonary disease: a review. Eur Respir J 2001; 17: 1024-1033.

22 Halm EA, Lee C, Chassin MR. Is volume related to volume in health care? A systematic review and methodological critique of the literature. Ann Intern Med 2002; 137: 511-520.
23 Burns LR, Wholey DR. The effects of patients, hospital and physician characteristics on length of stay and mortality. Med Care 1991; 29: 251-271.

24 Balmes JR. Occupational contribution to the burden of chronic obstructive pulmonary disease. J Occup Environ Med 2005; 47: 154-160.

25 Spurtzem JR, Rennard SI. Pathogenesis of COPD. Semin Respir Crit Care Med 2005; 26: 142-153. 